\title{
ULTRAVIOLET IMAGING POLARIMETRY OF THE LARGE MAGELLANIC CLOUD. II. MODELS
}

\author{
Andrew A. Cole, ${ }^{1,4}$ Kenneth Wood ${ }^{2} \&$ Kenneth H. Nordsieck ${ }^{1,3}$ \\ Draft version July 9, 2021
}

\begin{abstract}
Motivated by new sounding-rocket wide-field polarimetric images of the Large Magellanic Cloud Cole et al. 1999a), we have used a three-dimensional Monte Carlo radiation transfer code to investigate the escape of near-ultraviolet photons from young stellar associations embedded within a disk of dusty material (i.e., a galaxy). As photons propagate through the disk, they may be scattered or absorbed by dust. Scattered photons are polarized and tracked until they escape to be observed; absorbed photons heat the dust, which radiates isotropically in the far-infrared, where the galaxy is optically thin. The code produces four output images: near-UV and far-IR flux, and near-UV images in the linear Stokes parameters Q and U. From these images we construct simulated UV polarization maps of the LMC. We use these maps to place constraints on the star+dust geometry of the LMC and the optical properties of its dust grains. By tuning the model input parameters to produce maps that match the observed polarization maps, we derive information about the inclination of the LMC disk to the plane of the sky, and about the scattering phase function $g$. We compute a grid of models with $i=28^{\circ}, 36^{\circ}$, and $45^{\circ}$, and $g=0.64,0.70,0.77,0.83$, and 0.90 . The model which best reproduces the observed polarization maps has $i=36^{\circ}{ }_{-5}^{+2}$ and $g \approx 0.7$. Because of the low signal-to-noise in the data, we cannot place firm constraints on the value of $g$. The highly inclined models do not match the observed centro-symmetric polarization patterns around bright $\mathrm{OB}$ associations, or the distribution of polarization values. Our models approximately reproduce the observed ultraviolet photopolarimetry of the western side of the LMC; however, the output images depend on many input parameters and are nonunique. We discuss some of the limitations of the models and outline future steps to be taken; our models make some predictions regarding the polarization properties of diffuse light across the rest of the LMC.
\end{abstract}

Subject headings: polarization - methods: numerical - galaxies: individual (LMC) - ISM: dust, structure

\section{INTRODUCTION}

Polarimetric imaging provides a unique window on the 3 -dimenional stuture of astrophysieal ojects, and therefore on the physical processes operating in a wide range of stellar and interstellar environments. The most important processes giving rise to interstellar polarization are scattering by dust, and transmission through aligned dust grains.

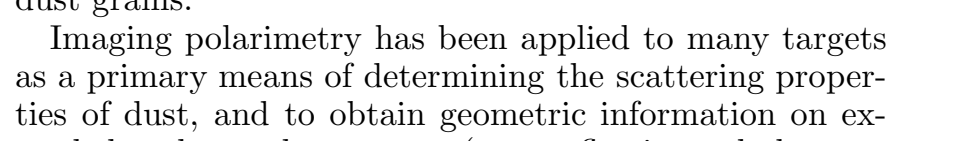

tended and complex sources (e.g., reflection nebulae, active galactic nuclei, and comets).

The vacuum ultraviolet is an especially favorable wavelength regime for these studies; polarimetric efficiencies are high, and polarized backgrounds are low (Nordsieck et al. 1993. . Moreover, a relatively small number of bright stars emit the majority of VUV photons, greatly simplifying the accurate tracing of source-scatterer-detector geometry over the case in the optical and near infrared.

The University of Wisconsin's Wide-Field Imaging Survey Polarimeter (WISP) was developed to obtain the first wide-field astronomical polarization images in the vac- uum ultraviolet. This rocket-borne instrument has been flown three times to date, providing high-quality images of reflection nebulosity in the Pleiades open cluster Gibson, Holdaway, \& Nordsieck 1995, Gibson 1997, Gibson \& Nordsieck 1999, in preparation), and photopolarimetry of Comet Hale-Bopp (Harris et al. 1997). Additionally, WISP obtained polarimetric images of the Large Magellanic Cloud (LMC); these observations represent the first wide-field, UV polarization images ever obtained (Nordsieck et al. 1996). Analysis of the LMC data (Cole et al. 1999a hereafter Paper I) found that the diffuse UV light is polarized at a 5-10\% level, consistent with starlight scattered by dust; that the strongest source of illumination in the WISP field is the H II complex N11; and that the UV starlight must account for most of the heating of diffuse dust in the LMC.

In this paper, we report on our program to model the WISP polarization maps of the LMC using a Monte Carlo radiation transfer code to constrain the optical properties and scattering geometry of the dust in the LMC's diffuse interstellar medium. $\S 1.1$ and $\S 1.2$, respectively, describe the observational results which motivate this work, and the interpretive issues addressed by our models.

In $\S 2$, we describe in detail the basic astrophysical ingre-

\footnotetext{
${ }^{1}$ cole@astro.wisc.edu; Department of Astronomy, University of Wisconsin-Madison, 475 North Charter St., 5534 Sterling Hall, Madison, WI, 53706 .

${ }^{2}$ kenny@claymore.harvard.edu; Smithsonian Astrophysical Observatory, 60 Garden Street, Cambridge, MA, 02138.

${ }^{3}$ khn@sal.wisc.edu; Space Astronomy Laboratory, University of Wisconsin-Madison, 1150 University Avenue, Madison, WI, 53706.

${ }^{4}$ Current address: Department of Physics \& Astronomy, University of Massachusetts, Amherst, MA 01003-4525.
} 
dients of our models, which are the distributions in space, size, and luminosity of the illuminating OB associations and scattering dust medium. In $\S 3$ we discuss the scattering and polarizing properties of the dust grains and the way in which we parameterize the total amount of dust present. $\S 4$ describes the Monte Carlo engine of our radiation transfer code, which is innovative in its ability to track the processing of near-UV photons into thermal IR radiation by heated interstellar dust grains. In $\S 5$ the modelling algorithm is explained, relating the procedure by which we explored parameter space for the "best" models. $\S 6$ presents the model images and polarization maps and the conclusions we can draw about the inclination of the LMC disk and the scattering asymmetry of its dust grains. We are careful to note the many shortcomings of this simple model, which nonetheless reproduces many of the observed near-ultraviolet and far-infrared properties of the LMC for a reasonable set of inputs.

\subsection{Observations: the Wide-Field Imaging Survey Polarimeter}

A $1.5 \times 4.8$ area of the western side of the LMC was observed with the rocket-borne Wide-Field Imaging Survey Polarimeter (WISP) on November 20, 1995. $4 \times 80$ second exposures in an intermediate-band, near ultraviolet filter $(\lambda=2150 \AA, \Delta \lambda=300 \AA)$ were used to create intensity and polarization maps of the field. The observations were centered at $\alpha=04^{\mathrm{h}} 59^{\mathrm{m}}, \delta-67^{\circ} 53^{\prime} \quad(\mathrm{J} 2000.0)$ and aligned roughly north-south. The WISP instrument is described in detail in Nordsieck et al. 1993; the reduction, calibration, and analysis of the LMC flight data are given in Paper I.

The minimum diffuse UV surface brightness, $5.6 \pm 3.1$ $\times 10^{-8} \mathrm{erg} \mathrm{s}^{-1} \mathrm{~cm}^{-2} \AA^{-1} \mathrm{Sr}^{-1}$, is larger than any known stray light background, and is clearly due to light originating within the LMC. The surface brightness of this diffuse UV background is correlated with areas of high $\mathrm{H} \mathrm{I} \mathrm{column}$ density and is linearly polarized at the $\sim 10 \%$ level. This suggests that reflected $\mathrm{OB}$ starlight contributes at least half of the LMC's diffuse UV background. The ISM of the Large Magellanic Cloud apparently acts as a kiloparsecscale reflection nebula in the near ultraviolet. Paper I found evidence for weak centro-symmetric scattering halos around some of the large OB complexes in the WISP field. The B2 complex (Martin et al. 1976), however, lacked such a halo; this was interpreted to mean that B2 is located either within an $\mathrm{H} \mathrm{I}$ hole or well above the plane of the LMC disk.

\subsection{Modelling Goals}

It is desirable to test the interpretation of Paper I; to this end we have undertaken to model the radiation transfer of ultraviolet photons from their origins in hot stars, through the dusty ISM of the LMC's disk, to Earth. Using these models we hope to determine whether or not the observed level of polarization is consistent with the reflection nebula interpretation. We also wish to determine the expected polarization pattern around B2 for a location within the disk; perhaps a non-detection of centro-symmetry is to be expected for this region.

Using a specialized Monte Carlo radiation transfer code, we set out to determine whether or not reasonable values for the dust optical depth, scattering geometry, and dust grain optical properties can account for the WISP observations. Under the assumption that the reflection nebula interpretation is correct, we can use the polarization properties of the model to place constraints on the dust properties and inclination of the disk of the LMC.

\section{MODEL INGREDIENTS AND ASSUMPTIONS}

\subsection{Illuminating Sources}

The perfect model of the Large Magellanic Cloud would incorporate the luminosity contributions of every field star and star cluster into its input parameters. This is obviously impractical, and so we must find a more tractable subset of objects with which to illuminate the LMC's dust. The star-formation rate of LMC field stars has been roughly constant for the past $\approx 1-2$ Gyr (e.g., Gallagher $e$ al. 1998; Westerlund 1997 and references therein). This recent activity has been accompanied by the formation of a large number of "blue populous" star clusters and OB associations; the young clusters of the LMC are both more frequent per unit field star mass and individually larger than their Milky Way counterparts (e.g., Elson \& Fall 1985; van den Bergh 1984).

Data from the UIT instrument suggests that $\approx 75 \%$ of the flux from the LMC at $\lambda=1500 \AA$ originates from stellar associations within the regions of nebulosity catalogued by Davies, Elliot \& Meaburn 1976 (Parker et al. 1998). For the western side of the LMC (observed by WISP), this interpretation holds true at $2150 \AA$. In the WISP image, most of the well-detected sources can be identified with OB associations (Lucke \& Hodge 1970), or open clusters younger than $\approx 200$ Myr. Clusters older than this, e.g., the massive 1 Gyr-old young globular cluster NGC 1783, are undetected in our image. Individual supergiants among the field stars (Sanduleak 1969) can be detected, but are minor contributors to the total observed flux.

We therefore chose to take as our illuminators the 122 OB associations of Lucke \& Hodge 1970, because a homogeneous dataset of ultraviolet photometry at two wavelengths exists for the entire sample (Smith et al. (1987), hereinafter $\mathrm{SCH}$ ). Due to the lack of a uniform sample of ultraviolet photometry, we have ignored the young open clusters in this first model; some of these clusters, e.g., NGC 1818, NGC 1755, and NGC 1711, contribute significant UV flux to the WISP image.

The positions of the $\mathrm{OB}$ associations from Lucke \& Hodge 1970 were transformed onto the model's rectilinear coordinate system at a scale of $15^{\prime}$ per grid unit. The scale was chosen in order to accomodate output images of the entire LMC, and the grid spacing is well-matched to the final, binned resolution of the WISP observations.

The origin of the coordinate system was chosen following Westerlund 1990 to lie at $05^{\mathrm{h}} 24^{\mathrm{m}},-69^{\circ} 50^{\prime}$ (B1950.0); this corresponds to the centroid of optical light in the galaxy (deVaucouleurs \& Freeman 1973). The distribution of OB associations in the LMC, as in the Galaxy, can be assigned some finite scale height above and below the galactic midplane. However, because this scale height is likely to be smaller than the scale height of dust (Harris et al. (1997)), and we have no a priori knowledge of the relative positions of each association along the line of sight, we have forced the illuminating sources in our models to lie in the plane of the LMC disk. OB associations are not point sources, 
having radii of $\approx 15-150 \mathrm{pc}$ (Lucke \& Hodge 1970); for simplicity, we have modelled them as spheres. The radii of our illuminators do not directly correspond to the optically defined dimensions of the Lucke \& Hodge associations, but were determined from the vacuum ultraviolet images of SCH.

SCH photometered the entire Lucke \& Hodge catalog at 1500 and $1900 \AA$, using rectangular apertures that were matched to each association by hand; their Table 1 gives the total area of each of their apertures. We assigned radii to our spherical sources by setting their projected surface areas equal to the areas given by SCH in their Table 1 . In just two cases (LH 15 and LH 77) did we deem the deviations from sphericality strong enough to warrant a more complex procedure. Both associations lie within supergiant shells on the northern side of the LMC disk. LH 15, within LMC-1, is contained within the field of view of the WISP CCD image; LH 77, at the center of LMC4 , is quite bright and resembles a quadrant of a circle's circumference. These two associations were broken up arbitrarily into four identical sub-associations, which more closely reproduced the visual appearance of these sources.

Near-UV luminosities were assigned to the sources based on the photometry of SCH at $1500 \AA\left(m_{15}\right)$ and $1900 \mathrm{~A}$ $\left(m_{19}\right)$. We dereddeded the SCH photometry and applied a correction for the difference in bandpass between their filters and the WISP filter at $2150 \AA$. The reddening values given by Lucke 1974 were broken down into LMC and foreground Galactic components; following $\mathrm{SCH}$, the maximum value of foreground reddening was taken to be $\mathrm{E}_{B-V}^{M W}$ $=0.07 \mathrm{mag}$. Any additional reddening towards the individual associations was attributed to dust within the LMC. Reddening values for each source were derived following the procedure outlined in Paper I, as were corrections for the differing bandpasses used by SCH and in Paper I.

The derived extinction values were found to be in good agreement with those published by Smith et al. 1990 in an erratum to $\mathrm{SCH}$. The bandpass corrections ranged from $-0.5 \mathrm{mag}$ to $+0.6 \mathrm{mag}$ for the 122 Lucke-Hodge OB associations. The corrected magnitudes were converted into monochromatic fluxes for the Monte Carlo photon generator using the standard relation $\mathrm{F}_{U V}=10^{-0.4\left(m_{0}+21.1\right)}$ $(\mathrm{SCH})$. The source positions, radii, and luminosities are given in an appendix to this paper, in Table A1.

\subsection{Dust Distribution}

The LMC is a disk galaxy and we have chosen to represent its dust density distribution using an exponential decay with radius and a hyperbolic secant law in height above the midplane (Binney \& Tremaine 1987):

$$
\rho(r, z)=A \exp \left(\frac{-r}{r_{d}}\right) \operatorname{sech}^{2}\left(\frac{z}{2 z_{d}}\right),
$$

where the constant $A$ is set by the optical depth of the model (see $\S 3$ below), and the dust scale length $r_{d}$ and scale height $z_{d}$ are taken from the literature. Observational estimates of the LMC's dust scale length were unavailable, and so we set $r_{d} \equiv 2.6 \mathrm{kpc}$ (12 grid units), the scale length of the old stellar population Kinman et al. 1991) 1.

The dust scale height, $z_{d}$, must also be estimated in-

${ }^{1}$ We have assumed a distance to the LMC of $50 \mathrm{kpc}$ directly. Harris et al. (1997) measured two reddeningfree photometric indices for $2069 \mathrm{O}$ and B stars in a 2.9 $\operatorname{deg}^{2}$ area centered $\sim 2.6$ northwest of the optical center of the LMC. Using the distribution of reddening values they found that the data could be well-matched by a vertical distribution in which the dust has a scale height equal to twice that of the OB stars. Assuming the OB stars to lie in an extremely flattened disk, with scale height $\approx 100 \mathrm{pc}$ (c.f., Oestreicher \& Schmidt-Kaler 1995 for Galactic OB stars), we choose a dust scale height of $\approx 200$ pc, or 0.96 grid units.

As a first-order deviation from the smooth, azimuthally symmetric model dusk disk, we placed nine low-density cavities into the model, corresponding to the supergiant shells of Meaburn 1980. These shells were identified by the enhancements of $\mathrm{H} \alpha$ emission around their perimeters and are also characterized by extremely low HI column densities. They are thought to be roughly cylindrical, and "open-topped" (Westerlund 1997), but in our models they are defined by simple spherical cavities of low optical depth. The cavities are placed in the midplane of our model galaxy, using the positions and sizes from Meaburn 1980. We assign a density to the cavities by defining the near-UV optical depth $\tau_{c}$ across the diameter of a cavity. $\tau_{c}$ was derived from photometric measures of the reddening, $\mathrm{E}_{B-V}$, of the OB associations lying within the boundaries of the supergiant shells. These lie in the range 0.00 $\leq \mathrm{E}_{B-V} \lesssim 0.12$ (Lucke 1974), less $0.07 \mathrm{mag}$ of foreground reddening; we also assumed that roughly half of the observed reddening towards the $\mathrm{OB}$ associations was due to material in the near neighborhood of the stars and hence not a contributor to the optical depth of the cavity as a whole. We adopted a "typical" $\mathrm{E}_{B-V}$ of $0.01 \mathrm{mag}$, and assumed the $\mathrm{OB}$ associations to lie at the center of the spherical cavities; applying an LMC extinction law for the model's $2150 \AA$ photons, we set $\tau_{c}=0.1$. The catalog of supergiant shell parameters is given in the Appendix, in Table A2; the cavities and illuminators are mapped out in Figure 1.

The inclination of the LMC disk to the plane of the sky remains a matter of some debate (Westerlund 1997). It has become clear that the east (30 Dor) side of the LMC is closer than the western (WISP field) side. As shown in Table 3.5 of Westerlund 1997, both the inclination $\mathrm{i}$ and position angle line of nodes $\Theta$ are known only to a precision of a few tens of degrees. Measurements of $\Theta$ scatter around a north-south line, and so we adopt $\Theta=180^{\circ}$ for simplicity. Geometrical methods applied to young and old stellar populations as well as neutral and ionized gas have yielded results varying between $25^{\circ} \lesssim \mathrm{i} \lesssim 48^{\circ}$. The expected magnitude and spatial variation of polarization depend strongly on the scattering geometry in the disk of the model galaxy, and so we consider three values for $\mathrm{i}$ in our models: $28^{\circ}, 36^{\circ}$, and $45^{\circ}$. The WISP field, along the west side of the LMC, is tilted away from the Earth: as the inclination increases, photons must traverse larger path lengths through the absorbing dust layer in order to escape and be seen.

\section{DUST PROPERTIES}




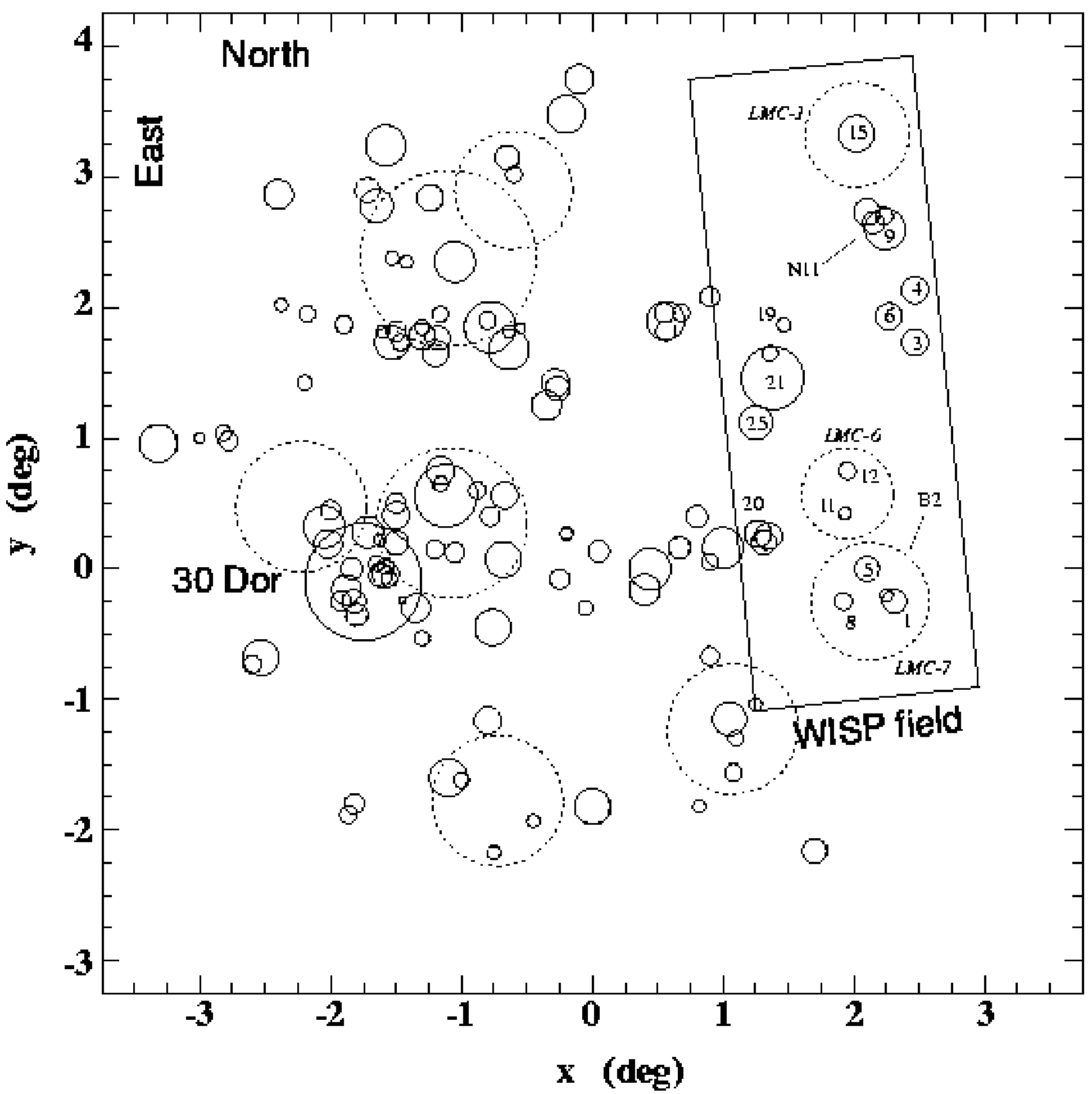

FIG. 1. - The distribution of illuminators (open circles) and cavities (dotted-line circles) in our model LMC. The positions of illuminators are taken from Lucke \& Hodge 1970, with radii and luminosities as described in $\$ 2.1$. Cavity positions are taken from Meaburn 1980. Positions in equatorial coordinates have been transformed onto a rectilinear grid with the origin at $5^{\mathrm{h}} 24^{\mathrm{m}},-69^{\circ} 50^{\prime}$ (B1950.0). OHfsets are given in degrees. North is up, East is to the left. OB Associations and supergiant shells in the WISP field (Paper I) are labelled with their Lucke-Hodge and Meaburn numbers, respectively. 
Along with the scattering geometry, the optical properties of interstellar dust grains control the linear polarization of stellar photons. Our models treat the scattering process using a standard Henyey-Greenstein phase function (Henyey \& Greenstein 1941), which depends on the albedo $a$, and asymmetry parameter $g$. The parameter $g$ defines the probability for an incident photon to scatter through an angle $\theta: \mathrm{P}(\theta) \propto \frac{\left(1-g^{2}\right)}{\left(1+g^{2}-2 g \cos \theta\right) \frac{3}{2}} \cdot g=$ 0 yields isotropic scattering, while $g=1$ gives pure forward scattering; $g<0$ corresponds to backscattering. To model the polarization, we follow White (1979) in the approximation $\mathrm{P}(\theta) \approx p_{\max } \frac{\sin ^{2} \theta}{1+\cos ^{2} \theta}$. $p_{\max }$ is the maximum polarization attainable in a single scattering event, for a scattering angle of $90^{\circ}$; the polarization of the scattered photon decreases for smaller and larger scattering angles.

The total amount of dust in the model is described by the single optical depth parameter $\tau_{e q} . \tau_{e q}$ is simply the optical depth of the model galaxy to a photon as it travels from center to edge through the midplane.

White (1979) tabulated the scattering properties of the Mathis et al. (1977) (MRN) Milky Way dust mixture. Deviations from the White (1979) values are to be expected for the LMC, which is in general more metal-poor than the Galaxy (Pagel et al. 1978; Dufour 1984). The LMC extinction curve shows a less pronounced $2175 \AA$ bump and a steeper rise into the far-UV than does the Galactic curve, attributable to variations in dust grain sizes and/or compositions (Nandy et al. 1981). Pei (1992) recalculated the albedo of Magellanic Cloud dust using an MRN grain-size distribution with the relative contributions from graphite and silicates scaled to match the observed mean extinction curves. We adopt the Pei (1992) value, $a=0.66$ for $\lambda=2150 \AA$, for all our models; this is $\approx 25 \%$ higher than the observed albedo of Milky Way dust (e.g., Witt et al. 1992). Pei (1992) did not include calculations for $g$ or $p_{\max }$ in his paper; we adopt the MRN-based value, $p_{\max }$ $=0.31$. Because the polarization of scattered starlight depends strongly on $g$, we compute families of models in which $g$ is permitted to vary.

In the ultraviolet, the phase function asymmetry parameter $g$ is poorly constrained by both models $(0.1 \lesssim$ $g \lesssim 0.7$; e.g., Murthy \& Henry 1994), and observations, $(0.3 \lesssim g \lesssim 0.9$; e.g., Sasseen \& Deharveng 1996), even for Milky Way dust. Variations in $g$ lead to differences in the expected polarization patterns, allowing us to infer its value through comparisons of the model output to observations. We chose to examine the moderately to strongly forward-throwing regime: $g=0.64$, corresponding to the MRN value, and $g=0.90$, suggested by observations of reflection nebulae in the Pleaides cluster (Gibson 1997). Intermediate values were chosen at $g=0.70,0.77,0.83$, close to the values derived by Witt et al. 1992 for the reflection nebula NGC 7023. The most recent models of the Pleiades nebulosity (Gibson \& Nordsieck 1999, in preparation) also indicate a moderate value for $g$.

For the LMC, the parameterization of dust mass using $\tau_{e q}$ is problematic, because the LMC is observed nearly face on and thus the derived value of $\tau_{e q}$ is strongly geometry-dependent. We derive an initial optical depth from observations of OB associations (Harris ed al. (1997)) , scaled by a geometric factor, and then cor- rect this value using the observed ultraviolet (Paper I) to far-infrared (DeGioia-Eastwood 1992) flux ratios. Harris et al. (1997) found a mean B-band optical depth through the face-on LMC $\tau_{B}=0.98 \times \cos$ i. Using the LMC mean extinction curve of Fitzpatrick 1986, the $2150 \AA$ optical depth to the LMC's midplane is $\tau_{U V}=\frac{1}{2} \times 2.4 \tau_{B}=1.2$ $\times$ cos i. The Harris et al. (1997) field is offset some 0.67 radial scale lengths from the centroid of our dust distribution, and so we adopt a pole-to-midplane central optical depth $\tau_{\text {pole }}=2.4 \times \cos$ i. $\tau_{e q}$ is finally obtained by scaling $\tau_{\text {pole }}$ by the flattening ratio of the disk. We adopt as our initial value:

$$
\tau_{e q} \equiv \tau_{p o l e} \frac{r_{d}}{z_{d}}=29.5 \cos i .
$$

This number was adjusted during the modelling procedure in order to match the observed ratio of WISP 2150 $\AA$ flux to IRAS $60 \mu \mathrm{m}$ flux (see $\S 5$, below).

\section{RADIATION TRANSFER}

We construct model scattered light images with a Monte Carlo continuum radiation transfer code which accounts for multiple photon scattering, and predicts the spatially resolved flux and polarization (Wood \& Jones 1997). In the radiation transfer calculation, the dust plus gas mixture has albedo, $a$, and a scattering phase function approximated by the Henyey-Greenstein phase function Henyey \& Greenstein 1941) with asymmetry parameter, $g$ (section 3). The code has been modified from the axisymmetric models of Wood \& Jones to include a three dimensional distribution of illuminating sources (section 2.1) this is crucial for modeling the UV scattered light pattern in the LMC. Additionally, we have added a new feature, where absorbed photons are not removed from the simulation, but "re-radiated" isotropically from the point of absorption to form a "far-infrared" image. This is a first approximation for predicting the far IR emission from our simulations. We are in effect assuming that all the UV emission that is absorbed is reradiated at one wavelength where the dust is optically thin. In order to compare our models to the WISP and IRAS images of the LMC, we must include additional factors that account for the fact that our "reprocessing" technique does not enforce radiative equilibrium (section 5).

\section{MODELLING PROCEDURE}

With the dust distribution and OB association properties held constant for all models, our goal is to find the combination of disk inclination and scattering phase function that best reproduces the observed polarization maps of Paper I. The polarimetric images of the LMC are the result of the interplay of a large number of physical processes and properties: the vertical, radial, and azimuthal dust distributions, the clumpiness of the dust, grain albedos, the distances above or below the midplane of each illuminating source, and the morphologies and densities of the supergiant shells all contribute to the observed polarization pattern.

By tuning all of these quantities independently in our models, we could create a model whose output reproduces the observations precisely. However, such a model would almost certainly be non-unique, and its physical reality 
would be highly questionable. We prefer instead to hold most of the model traits fixed and consider only the variation in the parameters that are expected to most strongly influence the polarization maps. For example, one of the observables we insist the models match is the ratio of ultraviolet to far-infrared light escaping from the galaxy. In reality, this obviously depends on the UV albedo $a$, but because $\tau_{\text {IR }} \ll 1$ and $\tau_{\mathrm{UV}}>1$, it also depends on the optical depth. We could trade off albedo and optical depth to tune this ratio precisely, but the model would suffer from severe non-uniqueness and could be driven to unphysical values of $a, \tau$, or both. Therefore we fix $a$ (see Section 3), and adjust $\tau$ until the model produces approximately the correct amount of infrared light.

We consider a grid of 15 models (see Table 1), comprising 3 values of disk inclination i, and 5 values for scattering phase function $g$. For each model, we perform an initial run in order to determine the correction to our first guess at $\tau_{e q}$. If the emitting dust is smoothly distributed and in radiative equilibrium, then the relation between absorbed starlight and thermal emission in the far-infrared depends simply on the dust optical depth. Hence we can attempt to match the amount of dust in our models to the true dust mass by a comparison of UV to IR fluxes; we correct our intial value of $\tau_{e q}$ by matching the model images in the UV and IR to the observed WISP $2150 \AA$ and IRAS 60 $\mu \mathrm{m}$ images.

Rather than resort to large-scale averaging over extended regions of the LMC, we choose instead to calibrate our model optical depths using one well-measured region that lies within the WISP field of view (Paper I): the N11 complex (also known as DEM 34 Davies, Elliot \& Me eburn 1976], B1 Martin et al. 1976], MC18 McGee, Brool s, \& Batchelor 1972|). N11 lies at the southern edge of the supergiant shell LMC-1 (Meaburn 1980), and contains a large $\mathrm{H}$ II region that is ionized by hot stars in $\mathrm{OB}$ associations LH9, LH10, LH13, \& LH14 Lucke \& Hodge 1970] This permits us to make accurate comparisons between our models and polarimetric observations.

The correction is complicated because of the mismatch between models and reality. From the models, we compare monochromatic stellar photons at $2150 \AA$ to a monochromatic far-infrared emission from dust which is heated by the starlight. From observations, we compare images in the $\lambda \approx 2150 \AA$ bandpass to emission at $60 \mu \mathrm{m}$; real dust is heated by starlight of all wavelengths. Therefore the model IR/UV ratio cannot be immediately compared to the observations. In order to find the appropriate dust optical depth, we require:

$$
\frac{\mathcal{F}_{\text {IRAS }}}{\mathcal{F}_{\text {WISP }}}=\varepsilon_{I R}^{U V} \eta^{U V} \eta^{I R} \frac{\mathcal{F}_{I R}}{\mathcal{F}_{U V}},
$$

where $\mathcal{F}_{\text {IRAS }}$ and $\mathcal{F}_{\text {WISP }}$ are the observed fluxes in the IRAS $60 \mu \mathrm{m}$ and WISP $2150 \AA$ bandpasses, and $\mathcal{F}_{I R}$ and $\mathcal{F}_{U V}$ are the fluxes in the model output IR and UV images. $\eta^{I R}$ is a correction factor to account for the fact that our model dust grains are not in thermal equilibrium; they radiate their absorbed energy at a single, average, far-infrared wavelength whose flux equals the bolometric
far-IR flux of the dust. $\eta^{I R}$ depends on the dust temperature and the wavelength dependence of the dust emissivity; we adopt the values from DeGioia-Eastwood 1992 in her calculation of the ionizing flux in N11.

$\eta^{U V}$ is a similar correction that relates the dust heating by photons in the WISP bandpass to the total dust heating from light of all wavelengths. We calculate $\eta^{U V}$ by integrating the light of N11 from $912 \AA$ to $3648 \AA$ using Kurucz model atmospheres, measurements of the initial mass function from DeGioia-Eastwood 1992 (also see Parker et al. 1998), and weighting the spectral energy distribution by an LMC extinction law.

$\varepsilon_{I R}^{U V}$ corrects for the fact that our dust grains are not in radiative equilibrium; the model re-emits one far-infrared photon for each absorbed near-ultraviolet photon; to conserve energy we must scale the model output images by the ratio of UV to IR mean photon energies, represented by $\varepsilon_{I R}^{U V}$.

To tune the optical depth of our models, we checked each output model against equation 3; where the model ratio exceeded the the observations, we lowered $\tau_{e q}$, and vice versa. By lowering the UV optical depth, we decreased the number of reflected photons that are subsequently absorbed, and hence the relative amount of far infrared emission. As a result of the tests, we adopted the parameters shown in Table 1 for each inclination; no systematic trend with $g$ was apparent, although the values of $\frac{\mathcal{F}_{I R}}{\mathcal{F}_{U V}}$ showed a scatter of $\pm 10 \%$ around the mean value at each inclination. Due to difficulties with the IRAS zeropoint calibration, and uncertainty in the WISP zeropoint, it is difficult to relate our model parameter $\frac{\mathcal{F}_{I R}}{\mathcal{F}_{U V}}$ to a physical flux ratio.

Because of the large number of photon sources, the number of output images, and the large number of pixels in each output image, large numbers of photons were required in order to obtain significant signal-to-noise to measure the polarization of the diffuse UV light. For each of the 15 permutations of $\mathrm{i}$ and $g$, we computed a "low" signal-to-noise model with $10^{8}$ photons propagating through the model galaxy. These models provided sufficient information to identify the models that matched the data well enough to merit a more detailed look.

We re-ran the best fitting model with $10^{9}$ photons in order to more accurately assess the mean level of polarization and track the variation in polarization level and position angle across the image. As we began to write up these results, we continued to let the model run in order to create the highest possible signal-to-noise in the output images.

\section{RESULTS \& DISCUSSION}

\subsection{Model Images \& Polarization Maps}

Our models produced as their output a set of four images for each run: the ultraviolet and infrared flux, and, in the ultraviolet, images of the linearly polarized flux Q and U. For analysis, the output data were converted into FITS format and examined within the IRAF ${ }^{2}$ suite of tasks.

Model images of the WISP-observed portion of the LMC are shown in Figure 2. From left to right, we show the model UV image, the WISP $2150 \AA$ image, the IRAS 60 $\mu \mathrm{m}$ image, and the model IR image. The far left and right

\footnotetext{
${ }^{2}$ IRAF is distributed by the National Optical Astronomical Observatories, which are operated by the Association of Universities for Research in Astronomy, Inc., under cooperative agreement with the National Science Foundation.
} 
TABLE 1

Assumed, Derived, and output parameters for our Monte Carlo models.

\begin{tabular}{ccccc}
\hline \hline$g^{\mathrm{a}}$ & $\mathrm{i}^{\mathrm{b}}(\mathrm{deg})$ & $\tau_{e q}^{\mathrm{c}}$ & $\langle p\rangle^{\mathrm{d}}(\%)$ & $\delta \mathrm{x}^{\mathrm{e}}(\operatorname{arcmin})$ \\
\hline 0.64 & 28 & 24.8 & $12.2 \pm 0.3$ & $0.6 \pm 0.6$ \\
0.64 & 36 & 22.4 & $12.4 \pm 0.3$ & $10.2 \pm 0.6$ \\
0.64 & 45 & 20.1 & $12.8 \pm 0.3$ & $16.2 \pm 0.6$ \\
0.70 & 28 & 25.9 & $12.7 \pm 0.3$ & $-0.6 \pm 0.6$ \\
0.70 & 36 & 23.3 & $12.6 \pm 0.3$ & $9.6 \pm 0.6$ \\
0.70 & 45 & 19.9 & $12.9 \pm 0.3$ & $15.6 \pm 0.6$ \\
0.77 & 28 & 25.5 & $12.9 \pm 0.3$ & $0.0 \pm 0.6$ \\
0.77 & 36 & 23.5 & $12.8 \pm 0.3$ & $8.4 \pm 0.6$ \\
0.77 & 45 & 19.8 & $13.0 \pm 0.3$ & $16.2 \pm 0.6$ \\
0.83 & 28 & 25.3 & $13.1 \pm 0.3$ & $-0.6 \pm 0.6$ \\
0.83 & 36 & 24.5 & $13.4 \pm 0.3$ & $6.6 \pm 0.6$ \\
0.83 & 45 & 20.3 & $13.2 \pm 0.3$ & $15.6 \pm 0.6$ \\
0.90 & 28 & 26.5 & $13.4 \pm 0.3$ & $-1.2 \pm 0.6$ \\
0.90 & 36 & 24.4 & $13.7 \pm 0.3$ & $7.2 \pm 0.6$ \\
0.90 & 45 & 20.8 & $14.1 \pm 0.3$ & $13.8 \pm 0.6$ \\
\hline \multicolumn{7}{l}{ Observations ${ }^{\mathrm{f}}$} & & $12.6 \pm 2.3$ & $8.4_{-5.4}^{+1.8}$ \\
\hline \multicolumn{5}{l}{}
\end{tabular}

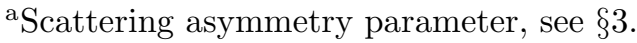

${ }^{\mathrm{b}}$ Inclination angle of LMC disk, see $\S 2.2$.

${ }^{\mathrm{c}}$ central optical depth in the plane.

${ }^{d}$ mean percentage of linear polarization.

${ }^{e}$ offset in polarization centers of symmetry, west of illuminating source.

${ }^{\mathrm{f}}$ from Cole et al. 1999a. 
panels of Figure 2 show the co-added results of multiple Monte Carlo runs, amounting to a total photon count of $4.1 \times 10^{9}$. Only a portion of the image is shown, to facilitate comparison to the WISP data (middle left panel of Figure 2). The dynamic range of the UV model is significantly smaller than the $2150 \AA$ data, because of the uniformly high optical depth in our models which results in nearly constant attenuation across the field. Many of the features are well-reproduced by the models, including the prominent $\mathrm{N} 11$ and $\mathrm{B} 2$ complexes which particularly dominate the IRAS $60 \mu \mathrm{m}$ image. The supergiant shells are less visible than expected in the model IR image; it lacks the "holes" visible in the $60 \mu \mathrm{m}$ data. However, their effect can be noted by comparison of the UV and IR appearance of the LH15 association at the upper right of the panels in Figure 2: LH15 is far brighter in the UV than the IR, a consequence of its location within the shell LMC-1 (see Figure 1).

Despite the obvious, and expected, lack of fine-scale structure in our model, we nonetheless are able to approximate the large-scale luminosity distribution in these two wavelength regimes. The most obvious failure of our model to reproduce small-scale structure in the dust distribution occurs at the association LH12 (see Figure 1). LH12 is an intrinsically bright association ( $\mathrm{SCH}$ ) that is highly reddened (Lucke 1974) and so appears faint to observers. However, LH12 lies within the LMC-6 supergiant shell; in our simple model, this drastically reduces the amount of obscuring dust along the LH12 sightline, causing us to dramatically overestimate the observed brightness of the association. A similar effect is discernible for the more southerly association LH5, highly reddened despite its position near the edge of the LMC-7 supergiant shell.

We created images of the degree of linear polarization, $p$, and position angle $\theta$ :

$$
\begin{aligned}
\mathrm{P} & =\left(\frac{\left(\mathrm{Q}^{2}+\mathrm{U}^{2}\right)}{\mathrm{I}^{2}}\right)^{0.5} \\
\theta & =0.5 \arctan \left(\frac{U}{Q}\right) .
\end{aligned}
$$

We created polarization maps with vector length proportional to $\mathrm{P}$ and position angle equal to $\theta$; our highest $\mathrm{S} / \mathrm{N}$ map is shown in Figure 3. The smooth dust distribution and finite number of illuminating sources account for the extreme regularity of the model polarization map.

In order to constrain the disk inclination of the LMC and the phase function $g$ of its dust, we compared the model polarization maps to those produced by Paper I from the WISP observations, shown side-by-side in Figure 4. The WISP observations, binned into $6^{\prime}$ pixels to increase signal-to-noise, showed some evidence for the presence of centro-symmetry about the brightest OB associations, but were hampered by the shortage of photons far from these bright regions; the effects of inhomogeneities in the scattering medium and a diffuse starlight component clearly dominate the appearance of the observed polarization maps.

Our points of comparison included the mean $\mathrm{P}$ and $\theta$, the shape of the high-polarization tail of $\mathrm{P}$, the degree of centro-symmetry around the brighest regions (N11 [B1] in North and N79 [B2] in the South), and the offsets of the polarization symmetry centers from the central OB associations. We also looked for variations in mean $\mathrm{P}$ and $\theta$ across the $1.5 \times 4.8$ field of view, but found no statistically significant differences to distinguish the various models. A

The comparison was not straightforward because of the systematic effects which dominate the errors in the observed polarization maps. In particular, the mean value of $\mathrm{P}$ was determined quite carefully: the WISP maps have been cleaned of marginal polarization detections where $\mathrm{P}$ $<2.5 \sigma_{\mathrm{P}}$, and become incomplete for polarizations less than $10 \%$. Therefore we applied a linear incompletess correction to the models, such that the model polarizations are weighted progressively less between $10 \%$ and $4 \%$, and all polarizations smaller than $4 \%$ are ignored. This mimics the observational bias towards detection of regions of high polarization.

The observed polarization maps, biased by incompleteness toward the detection of high polarization regions, showed a much higher scatter in polarization values than did the models. The observations also show a patchier distribution of $\mathrm{P}$; pixels with polarizations in the $5-10 \%$ range are frequently juxtaposed with $\approx 20 \%$ polarized regions. This indicates that the effects of small, optically thick clumps in the ISM are strongly influencing the scattered light component of the diffuse UV background. However, when we binned the models to the resolution of the observations and corrected for incompleteness at $\mathrm{P}<10 \%$, our models were consistent with the observed mean level of polarization across the WISP field. Scattered light from OB associations is indeed likely to account for a large fraction of the diffuse ultraviolet background in the LMC.

\subsection{Disk Inclination and the Dust Scattering Phase Function}

We found that our model polarization maps were sensitive to variations in $g$ and i; see Figure 5 . As $g$ increased from 0.64 to 0.90 , the mean polarization $\langle\mathrm{P}\rangle$ increased from $\approx 12.5 \%$ to $\approx 13.7 \%$. The disk inclination manifested itself most noticeably in the distribution of polarization vectors around bright sources, e.g., N11. For a face-on disk of scattering material, the polarization vectors form a centro-symmetric pattern about the illuminating source; the inclination introduces an asymmetry which shifts the center of the distribution away from the illuminator, perpendicular to the disk's line of nodes. As the inclination was increased, the offset in symmetry center of the polarization patterns (hereafter referred to as $\delta \mathrm{x}$ ) varied from 0.36 eastward, at $28^{\circ}$, to 16.2 westward, at $45^{\circ}$. These results are summarized in Table 1, and plotted in Figure 6 , in which $\langle\mathrm{P}\rangle$ and $\delta \mathrm{x}$ are plotted for each model. We have also plotted $\langle\mathrm{P}\rangle$ and $\delta \mathrm{x}$ for the observed polarization map, with the associated error bars. $\delta$ x tends to zero at inclinations of $\lesssim 30^{\circ}$ due to the competing effects of the disk inclination and the radial drop in dust density from east to west across the field; a plane parallel slab of scattering dust would show $\delta \mathrm{x}=0$ only for $\mathrm{i}=0$.

Interpolating in inclination, and adopting the uncertainty in our observational determination of $\delta \mathrm{x}$ from the binned WISP data, we find that the LMC's disk is inclined at $36^{\circ+2}$ to the plane of the sky. Insofar as the western side of the LMC resembles our models, this is a direct, geometric determination of its inclination. Although inconsistent with recent kinematic determinations (e.g., Kim 


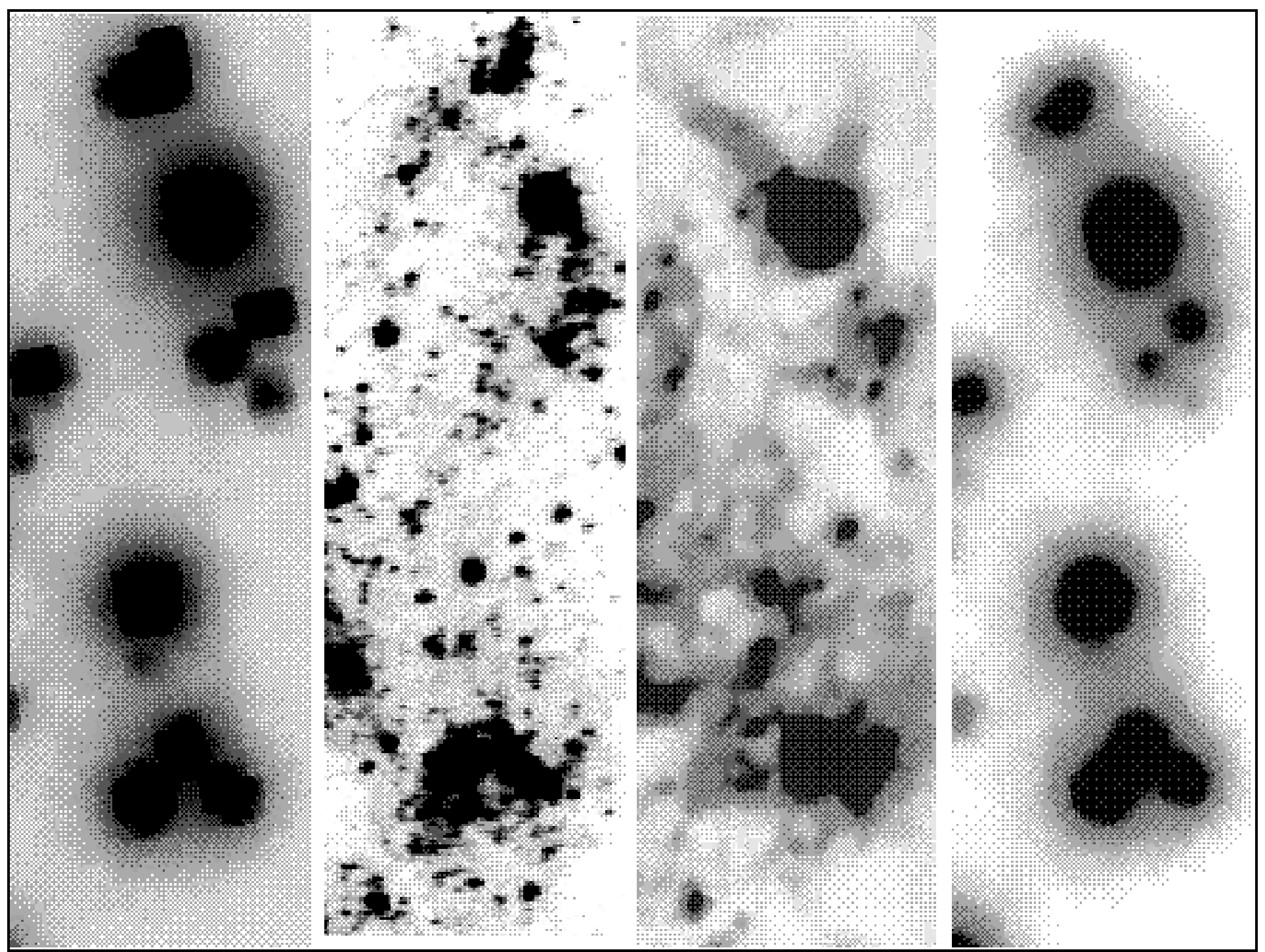

FIG. 2.- Model output images for $\mathrm{i}=36^{\circ}, g=0.70$, and $4.1 \times 10^{9}$ total photons. From left to right: model UV image; WISP $2150 \AA$ image (Paper I); IRAS $60 \mu \mathrm{m}$ image (Schwering 1989); model IR image. North is up, East is to the left. The differences between model and observations are attributable to the highly non-uniform distribution of stars and dust in the LMC. 


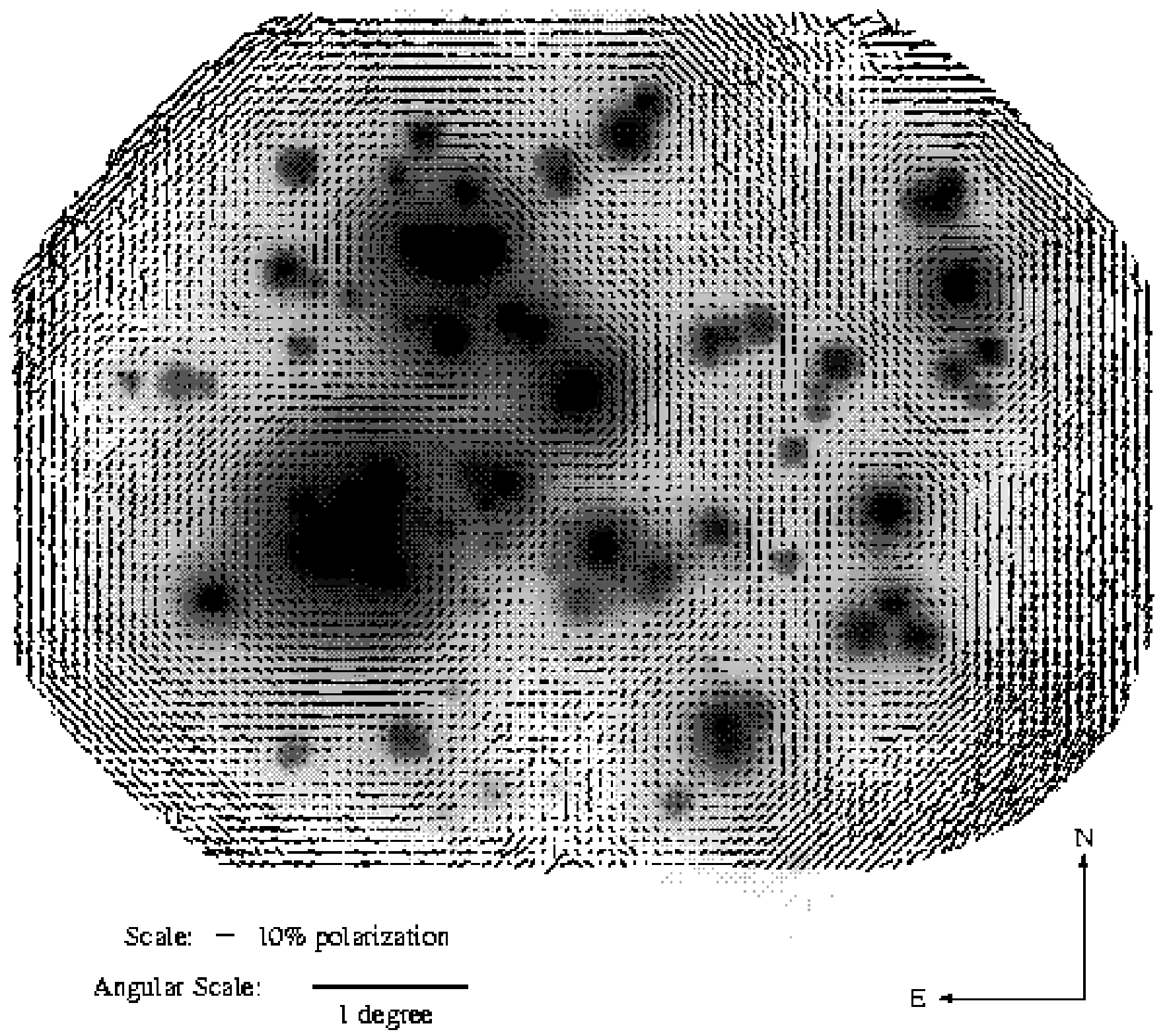

FIG. 3.- Model UV image with the polarization vectors derived from the Stokes Q and U model images overplotted. Because the only illumination derives from the $\mathrm{OB}$ associations, and the dusty scattering medium is smoothly distributed, the polarization vectors present clear, regular centrosymmetric patterns about the illuminators. North is up, east is to the left. 

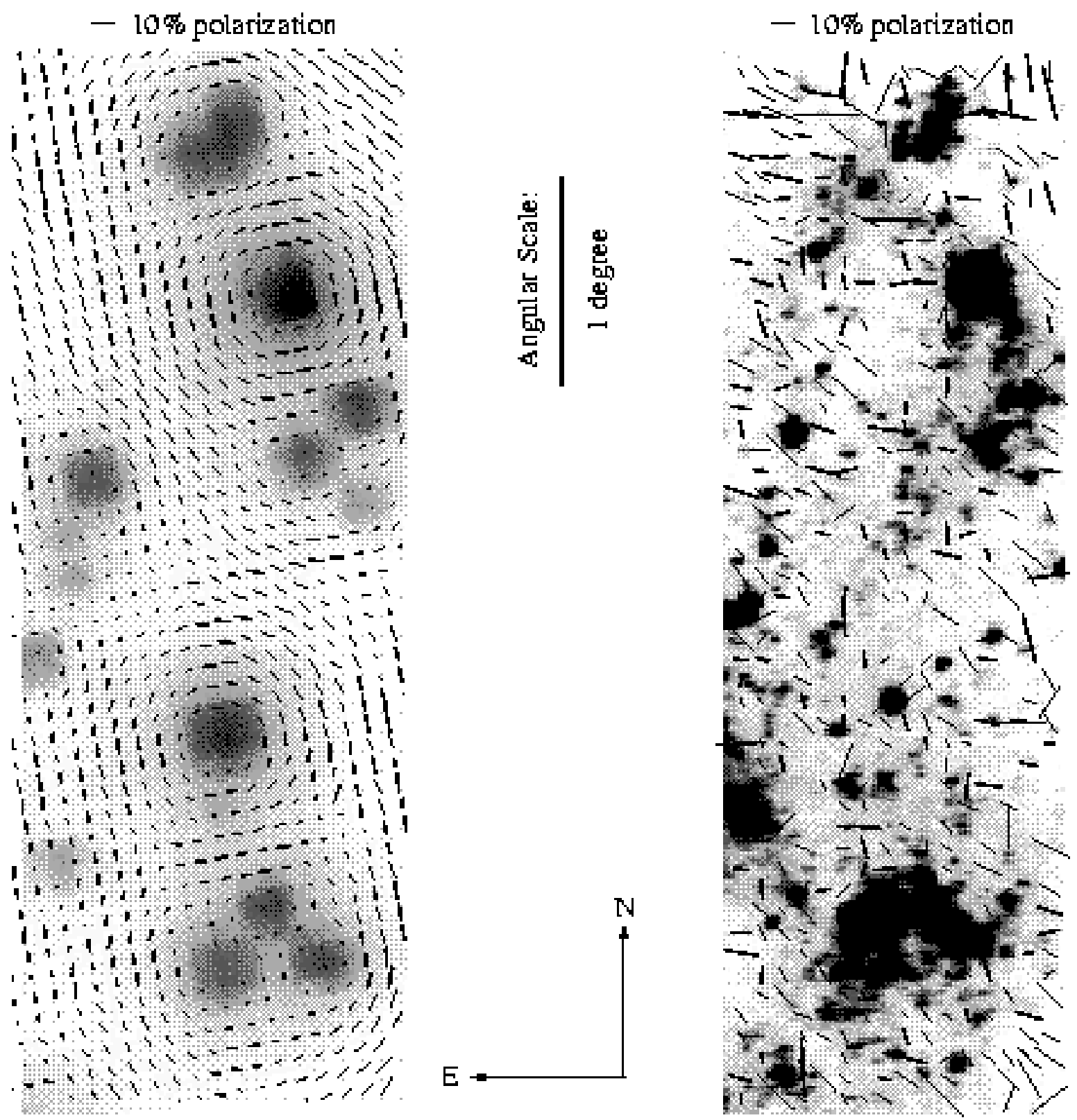

FIG. 4.- left: model UV image with polarization vectors overplotted; right: WISP $2150 \AA$ image with polarization vectors. The polarization and angular scales are shown in the figure. 


\section{WISP}

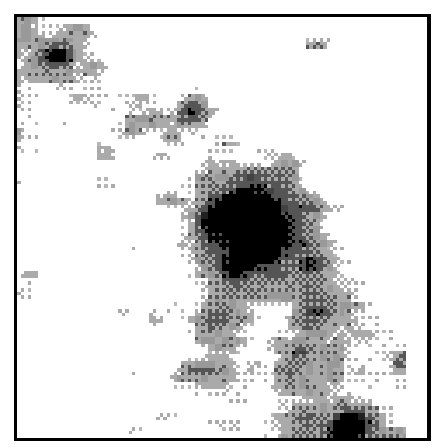

$g=0.64$

$$
i=28^{\circ}
$$

$i=36^{\circ}$

$i=45^{\circ}$
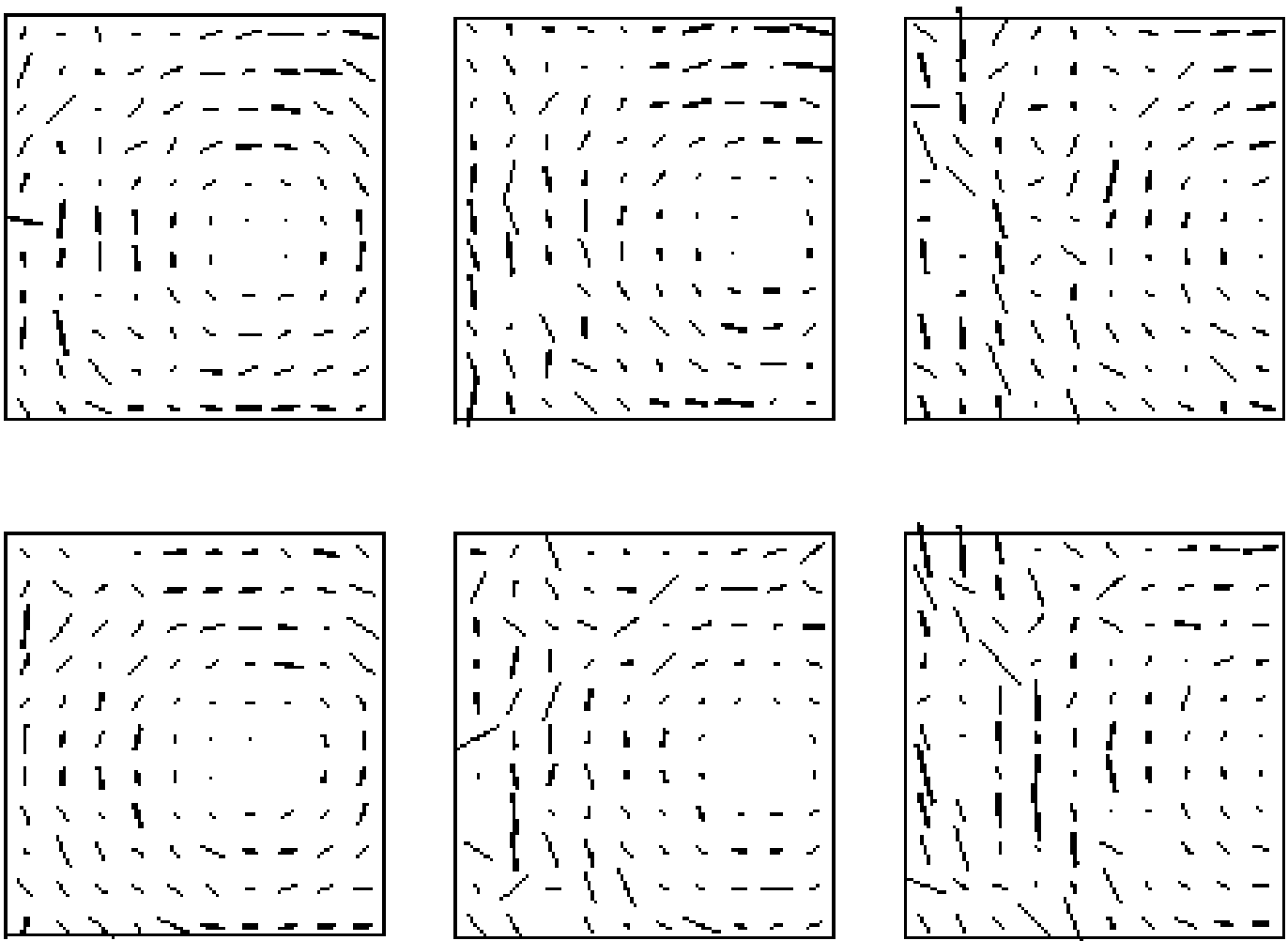

$$
g=0.77
$$
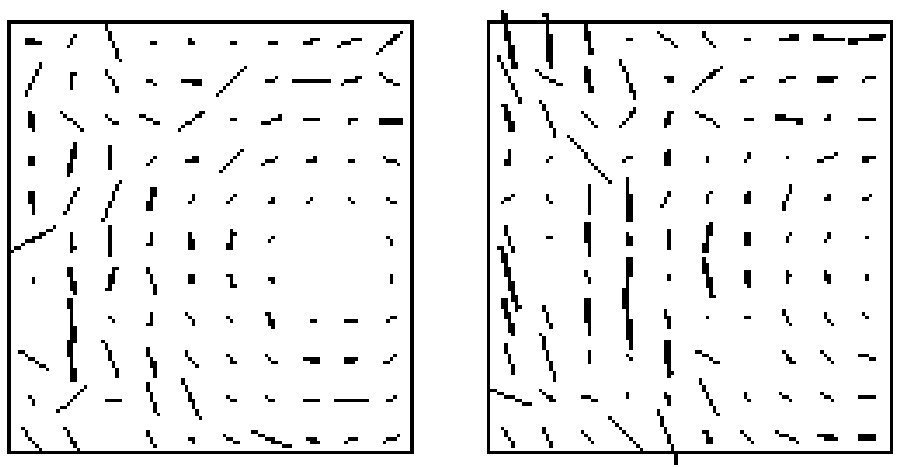

$$
g=0.90
$$

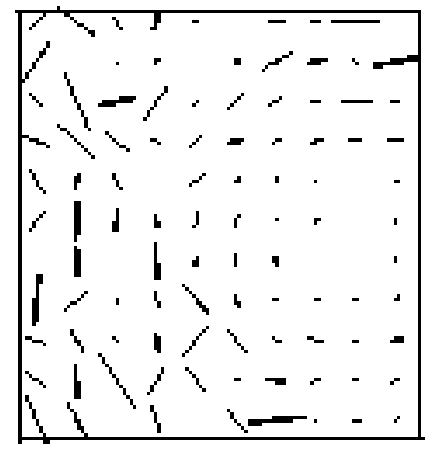

FIG. 5.- Model polarization maps for the area surrounding the H II complex N 11 demonstrate the dependence on inclination and phase function asymmetry. The actual $2150 \AA$ image of the region, $1^{\circ}$ on each side, is shown at upper left. Polarization maps for the same area are shown, arrayed by inclination and $g$ value. The smallest polarization vectors plotted are $1 \%$, the largest $35 \%$; observed values around $\mathrm{N} 11$ range from 0 to $\approx 10 \%$. 
et al. (1998), who found $\mathrm{i}=22^{\circ} \pm 6^{\circ}$ ), it is squarely in agreement with the average of reliable values tabulated by Westerlund 1997. If the dust scale height is larger than we have assumed, then the inclination required to yield a given $\delta \mathrm{x}$ is reduced; a significant change in $z_{d}$ would be required due to the concentration of scattering dust close to the midplane of the disk.

It is more difficult to draw conclusions regarding $g$. As seen clearly in Figure 6, the effect of high $g$ is mainly to increase the mean level of polarization. This ran counter to our expectations, for a high value of $g$ should produce smaller scattering angles, on average, resulting in polarizations much smaller than $p_{\max }$. The counterintuitive result is due to our simulation of the observational biases in the data of Paper I (see section 6.1); the high $g$ models did initially show lower levels of polarization, but were cleaned more severely by our rejection of low $\mathrm{P}$ pixels.

The low signal-to-noise of the WISP data and the larger than expected polarization fluctuations result in uncomfortably large errors in our determination of $\langle\mathrm{P}\rangle$. At the $3 \sigma$ level, we cannot rule out any value of $g$ between 0 and 1 . At the $1 \sigma$ level, we find $g=0.7 \pm 0.3$. The simple comparison of $\langle\mathrm{P}\rangle_{\text {mod }}$ to $\langle\mathrm{P}\rangle_{\text {obs }}$ throws away information about the distribution of $\langle\mathrm{P}\rangle$ within the images: we found that for $g$ $=0.83-0.90$, our models exhibited a strong high-P tail extending to $\approx 30 \%$. This tail was weak in the observations, and suggests that such high values of $g$ are less likely than would be inferred from the formal error distribution. However, as noted in Paper I, the highest polarization pixels typically show low flux values, and hence relatively large errors in both $\mathrm{P}$ and $\theta$. Low signal-to-noise might therefore introduce a bias against the high- $g$ models.

Our determination of $g$ is of comparable precision to values for the diffuse Milky Way dust, e.g. Murthy \& Henry 1994 and Sasseen \& Deharveng 1996; because of the complex scattering geometry it is far less precise than determinations based on Galactic reflection nebulae, e.g., Witt et al. 1992. Our value depends critically on the smoothness of the dust distribution as well as the relative positions along the line-of-sight of illuminators and scatterers (Witt \& Gordon 1996, Gibson \& Nordsieck 1999, in preparation). Also, as shown in Figure 2, the true distribution of ultraviolet luminosity in the LMC contains a non-negligible component due to stars not in the LuckeHodge associations. This additional source of direct, unpolarized, light would dilute the scattered light and hence reduce $\langle\mathrm{P}\rangle$ if included in the models. In such a model, higher values of $g$ would be required to account for the observed level of polarization.

\subsection{Individual associations}

Paper I lists nine UV-bright regions which appear to support scattering halos with the expected centrosymmetric pattern; we explore here the degree to which the simple model is able to reproduce these features of the polarization map.

\subsection{1. $N G C$ 1755, NGC 1711, N 186}

Two open clusters and a modest-sized star-forming region, these objects show some evidence of scattering halos; however, they are not included in our models.

\subsection{2. $L H 15, N 11, L H 12$}

These bright OB associations support scattering halos. The models reproduce them quite strongly. The northern associations LH 15 and N11 dominate the local UV radiation field, and are well detected in the WISP observations. LH 12 is far brighter in the model than is observed, a consequence of the strong deviation from smoothness of the surrounding dust structures (see §6.1). The observed scattering halo around LH 12 is quite weak, indicative of the contribution of increasing field star density in the southern half of the WISP field (Paper I).

\subsection{3. $\mathrm{LH}$ 4, LH 25, LH 16-17-20}

These regions show very weak evidence of scattering halos in the WISP image. In the models, there is little apparent indication of centro-symmetry about these associations. We note that each of these associations lies very close to the edge of the WISP image. Moreover, they lie near or within the scattering halos of brighter associations. These must be considered marginal detections.

\subsubsection{B2}

The B2 complex is made up of associations LH 1, 2, 5, and 8; its large $\mathrm{OB}$ star population led us to expect the presence of a strong centro-symmetric scattering halo. To the contrary, the paper I analysis of the WISP observations found no such halo. Possible explanations were suggested: the location of $\mathrm{B} 2$ above the plane of the dust layer, location of B2 within a large $\mathrm{H}$ I hole, or a possible bias against detection of scattering halos larger than $\approx 40^{\prime}$. Our model polarization maps show a centro-symmetric halo around B2; however, comparison to the comparable associations N11 and LH 15 showed it to be weaker than the halos of the northern associations. This is due, in the models, to the extnded (non-point-source) size of B2, as well as the contribution of light from LH 12 and other associations beyond the WISP field of view. In addition, the observations are degraded by the presence of high field star density in the southern WISP field, as well as the existence of a significant hole in the $\mathrm{H}$ I distribution (S. Kim, private communication). Part of this $\mathrm{H} I$ hole corresponds to the $\mathrm{H} \alpha$ supergiant shell LMC-7 (Meaburn 1980), which coincides with LH 8. We conclude that the Paper I nondetection of a scattering halo around B2 is astrophysical and not due to bias in their analysis, but the placement of the complex above the plane of the dust is not required by the observations.

\subsection{Limitations \& Future Work}

This suite of models represents our first attempt to model the radiation transfer of polarized light through a galaxy from a large number of discrete sources within a non-uniform dust layer. These models are able to reproduce the general morphology of ultraviolet and infrared images of the LMC; taken together with observed polarization data, they yield astrophysically interesting constraints on its inclination, and show consistency with dust properties expected from observations of the Milky Way.

However, the models provide a greatly simplified picture of the true structure of the LMC. Future adaptations of the radiation transfer code will address many of the simplifications; for others, additional observational material is required in order to refine our input parameters. A 


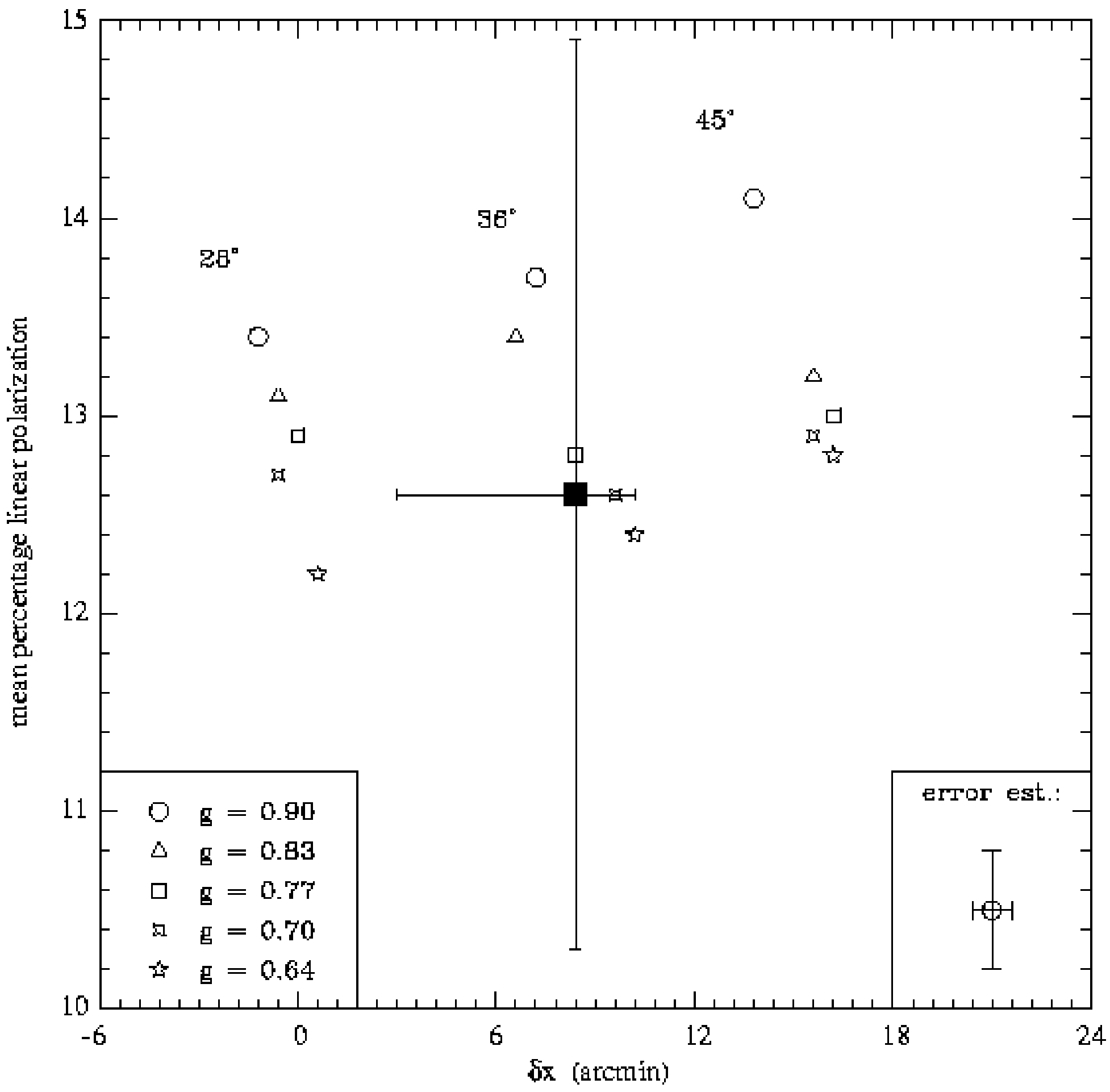

FIG. 6.- Model images created with differing values of $i$ and $g$ separate themselves in a plot of mean percentage of polarized flux against the offset between the observed scattering halos and illuminating sources. $\delta \mathrm{x}$ is shown in arcminutes. The solid square shows the observational data with errorbars (Paper I). A typical errorbar for the model-derived points are shown in the lower right corner. Each nearly vertical column of symbols is labelled with the disk inclination; the legend relating symbol type to $g$ is at lower left. 
more advanced version of the Monte Carlo code (dubbed "galaxy on a grid"), will allow the specification of dust and luminosity density at each individual point in the model grid; this flexibility will permit the exploration of arbitrarily complex geometries in future work.

The most severe drawback of our model is the adoption of a smooth dust distribution that lacks optically thick clumps. As noted by earlier authors (e.g., Witt, Thronson \& Capuano 1992; Witt \& Gordon 1996), the presence of small-scale, dense knots of absorbing material can greatly alter the emergent spectral-energy distribution of a galaxy's light. In this case, inferred values of $g$ are incorrect, and our ability to distinguish dust optical properties from the scattering geometry is lost (Gibson 1997, Gibson \& Nordsieck 1999, in preparation). Since the distribution of small-scale dust knots in the LMC is unknown, we have little recourse but to adopt a relatively homogeneous distribution of dust. The obvious effect of this approximation is that our models lack the large polarization fluctuations seen in the observations, presumably attributable to the presence of the patchy distribution of optically thick dust clouds.

A related drawback is the unknown line-of-sight distribution of illuminators relative to the dust. As shown by Witt, Thronson \& Capuano 1992, the relative amounts of scattering and absorption depend sensitively on where the illuminating sources lie relative to the dust. Along the same lines, we have modelled the supergiant shells as spheres, whereas evidence suggests they more closely resemble cylinders. One obvious effect of this mismatch is the presence of a foreground "haze" of IR emission above the shells in our models, which is not present in the IRAS data (see Figure 2).

The dust scale length in our models is highly uncertain; if we had chosen to adopt the scale length of H I inferred from the maps of Kim et al. (1998), our derived $r_{d}$ would have been some $40 \%$ smaller than the value we used. This in turn would have required a higher $\tau_{e q}$ in order to reproduce the UV-IR color of the diffuse light at the position of the WISP observations. Future models, using the galaxy on a grid system, would be set up to more closely match the complex column-density variations seen in the $\operatorname{Kim} e t$ al. (1998) H I maps, and therefore obviate the necessity of parameterization using $r_{d}$.

Although our scattering medium is unrealistically smooth, our stellar sources suffer from the opposite problem: we have considered only emission from large OB associations, ignoring the contributions of young, massive, open clusters and the field star population. This yields a model with a very highly clumped luminosity distribution; because our models ignore the direct stellar contribution to the diffuse ultraviolet light, they will tend to produce higher levels of polarization that would otherwise be observed. For this reason, our predicted value of $g$ is likely to be skewed towards lower values than a more complex model would produce. In future work, it will be desirable to add a smoothly varying component to the starlight, taken for example from the large-scale maps of MaucheratJoubert et al. 1980 .

\subsection{Predictions}

Our models extend over the central $\approx 10^{\circ} \times 10^{\circ}$ of the Large Magellanic Cloud, roughly 14 times the area covered by the WISP observations. This allows us to predict the general pattern of polarization that might be seen across the face of the LMC at near-ultraviolet wavelengths. Our models have been tuned to reproduce the optical depths and polarizations of the WISP field; a test of their validity and generality would be a comparison of our predictions to future ultraviolet polarimetric datasets across rest of the Large Cloud.

In Figure 7, we show the ultraviolet polarization vectors from our best model for the entire LMC. An optical image of the galaxy (Sandage 1961) is plotted to provide orientation and scale. If our model is valid, Figure 7 should predict the pattern of polarization across the face of the LMC. The predictions of the model are most uncertain in the region of the bar, which contains a high-surface brightness, intermediate-age population of field stars and numerous young open clusters not acounted for in our models (see, e.g., Hodge \& Wright 1967). We comment upon some regions of interest that may be likely future targets for polarimetric study.

We see that the 30 Dor region itself shows low levels of polarization, although it is responsible for much of the scattered light within a kiloparsec or more. The bar is expected to show low levels of polarization $(\lesssim 5 \%)$, with most of that due to 30 Dor at the eastern end. The bright H II region N51 (north of the central bar) should produce a centrosymmetric pattern similar to that of N11 (in the WISP field), although it will be weakened on the eastern and southern sides by radiation from 30 Dor. Finally, the southern spiral arm seen in the H I maps of Kim et al. (1998), beyond the southern limit of Figure 7 , is predicted to scatter light from as far north as 30 Doradus, giving rise to faint diffuse light that is polarized at the $20-30 \%$ level. It is expected that these models overpredict the mean levels of polarization because of the neglected contribution of direct light from field stars.

\section{SUMMARY}

Using a Monte Carlo radiation transfer code, we have modelled the observed ultraviolet polarization maps of the LMC obtained with the WISP instrument. Our code follows the tracks of stellar photons from their origins within OB associations, through a smoothly distributed exponential dust disk containing low-density cavities. By accounting for far-infrared thermal emission from heated dust, we are able to parameterize the total amount of dust present by its optical depth.

Dust-scattered starlight gives rise to linear polarizations; the magnitude and position angle of the polarization vectors allow us to derive information regarding the scattering geometry of stars+dust within the LMC and the optical properties of Magellanic Cloud dust. We consider three disk inclinations between $28^{\circ}$ and $45^{\circ}$, and five values for the phase function asymmetry parameter $g$, between 0.64 and 0.90 . We derive:

- The inclination of the disk of the LMC to the plane of the sky is $36^{\circ+2}$. This is in agreement with other results (Westerlund 1997), but not with a recent kinematic determination (Kim et al. (1998)). Our determination contains a dependence on the dust scale height; we have assumed $z_{d}=200 \mathrm{pc}$, but smaller values would imply larger inclinations, and vice versa.

- The most likely value for $g$ of 0.70 ; the uncertainty in 


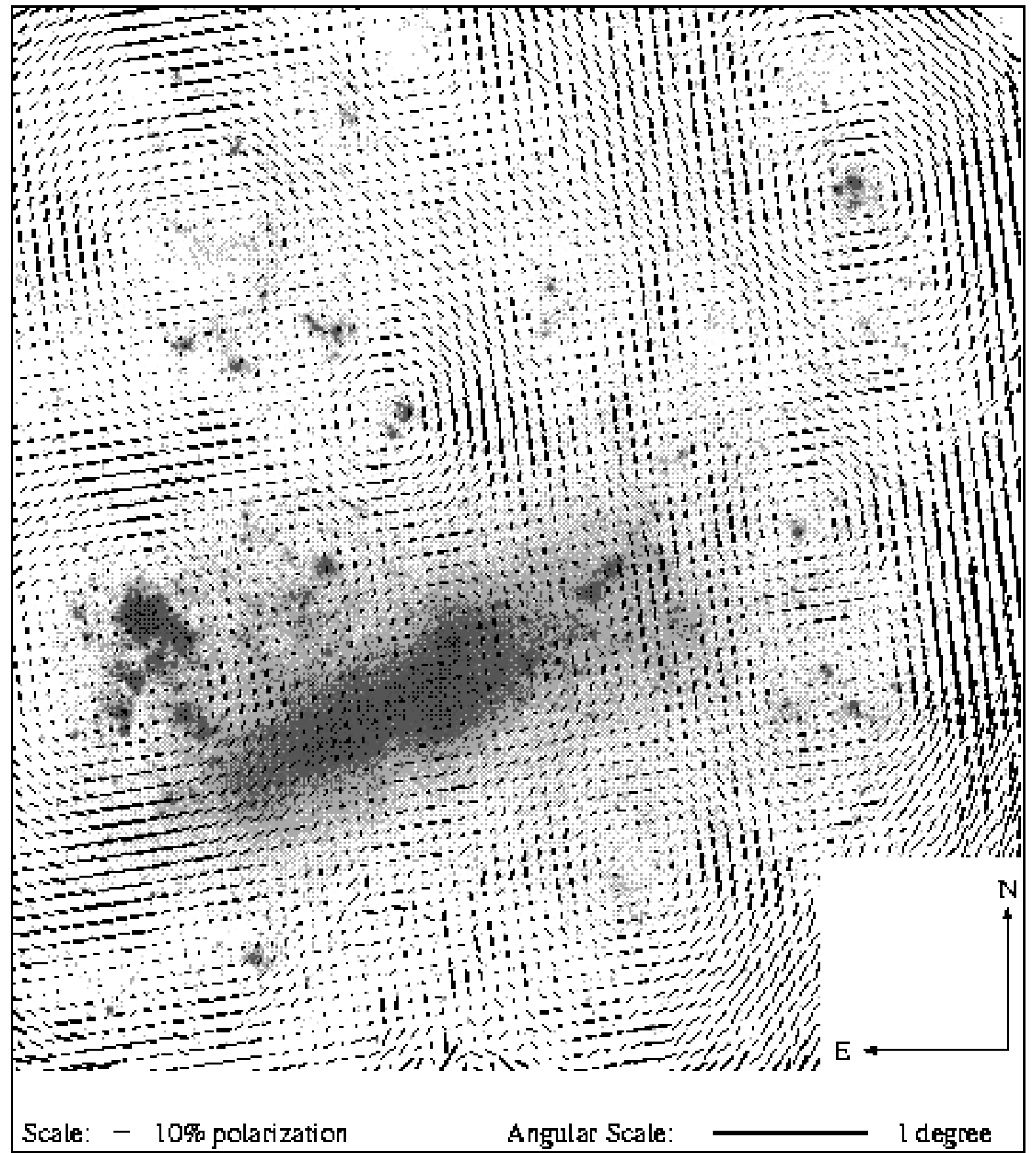

FIG. 7.- Polarimetric predictions for the LMC: our model UV polarization vectors are overlain on an optical image $(\lambda \approx 6600 \AA)$ of the galaxy from Sandage (1961). The WISP field of view is at the right. North is up, East is to the left. 
the observations does not permit us to rule out any value for $g$. Higher signal-to-noise data would sharpen our estimate, but a more precise method for estimating $g$ from our models is needed as well. For $g$ above 0.77 , the models produce a higher fraction of highly polarized $(p \gtrsim 20 \%)$ pixels than are observed. However, the neglect of direct light from field stars probably leads us to understimate $g$. Our value $g \approx 0.7$ is consistent with values derived from ultraviolet surface photometry of the Galactic reflection nebula NGC 7023 (Witt et al. 1992).

- Our best model predicts that scattered light from 30 Doradus dominates the eastern side of the LMC's diffuse UV radiation field; the presence of this "mini-starburst" region may be felt as far south as $-72^{\circ}$. Data for this region would be of great value in constraining the LMC's structure and geometry.

Our models are the first attempt to replicate the observed images and polarization maps of the Large Magellanic Cloud. It is encouraging that our model is able to approximately match the observations of Paper I for a reasonable set of input parameters; we do not expect that we have found a unique solution for i, $g$, or the geo- metric parameters that go into the model. The modelling procedure should be applicable to more general problems in the interpretation of galaxy polarization maps. However, a large number of simplifying approximations and assumptions have been made. The most serious of these is the smoothness of the adopted model dust distribution. Future work will be able to take into account the complexity of the LMC's H I distribution, which is dominated by flocculent spiral arms, supergiant shells, and small scale filaments (Kim et al. (1998)).

WISP is supported by NASA grant NAG5-647. K.W. acknowledges support from NASA's Long Term Space Astrophysics Research Program (NAG5-6039). This modelling project began as a simple exercise in radiation transfer with Joe Cassinelli in the spring of 1996 and has continued to grow from there. A.A.C. would like to thank Joe Cassinelli and Jay Gallagher for their patience and encouragement during the intervening years. We would like to thank the anonymous referee for suggestions which significantly improved the clarity of this paper.

\section{REFERENCES}

Binney, J., \& Tremaine, S. 1987, Galactic Dynamics, (Princeton Univ. Pr., Princeton).

Cardelli, J.A., Clayton, G.C., \& Mathis, J.S. 1989, ApJ, 345, 245.

Cole, A.A., Nordsieck, K.H., Gibson, S.J., \& Harris, W.M. 1999, AJ, in preparation (Paper I).

Davies, R.D., Elliott, K.H., \& Meaburn, J. 1976, MmRAS, 81, 89.

DeGioia-Eastwood, K. 1992, ApJ, 397, 542.

deVaucouleurs, G., \& Freeman, K.C. 1973, Vistas Astron., 14, 163.

Dufour, R.J. 1984, in IAU Symp. No. 108, eds. S. van den Bergh, K.S. deBoer, (Dordrecht: Reidel), p. 353.

Elson, R.A.W., \& Fall, S.M. 1985, ApJ, 299, 211.

Fitzpatrick, E.L. 1986, AJ, 92, 1068.

Freeman, K.C., Illingworth, G., \& Oemler, A., Jr. 1983, ApJ, 272, 488.

Gallagher, J.S., III, Cole, A.A., Holtzman, J.A., \& Smecker-Hane, T.A. 1998, in IAU Symp. 190, New Views of the Magellanic Clouds, in press.

Gibson, S.J., Holdaway, M.A., \& Nordsieck, K.H. 1995, BAAS, 27, 1349.

Gibson, S.J. 1997, BAAS, \#191, \#32.01.

Harris, J., Zaritsky, D., \& Thompson, I. 1997, AJ, 114, 1933.

Harris, W.M., Nordsieck, K.H., Scherb, F., \& Mierkiewicz, E.J. 1997, DPS, 29 , \#32.08.

Henyey, L.G., \& Greenstein, J.L. 1941, ApJ, 93, 70.

Hodge, P.W., \& Wright, F.W. 1967, The Large Magellanic Cloud, (Smithsonian Pr.: Washington).

Kim, S., Staveley-Smith, L., Sault, R.J., Kesteven, M.J., McConnell, D., \& Freeman, K.C. 1997, PASA, 14, 119.

Kim, S., Staveley-Smith, L., Dopita, M.A., Freeman, K.C., Sault, R.J., Kesteven, M.J, \& McConnell, D. 1998, ApJ, 503, 674.

Kinman, T.D., Stryker, L.L., Hesser, J.E., Graham, J.A., Walker, A.R., Hazen, M.L., \& Nemec, J.M. 1991, PASP, 103, 1279.

Kurucz, R.L. 1991, in Precision Photometry: Astrophysics of the Galaxy, edited by A.G.D. Philip, A.R. Upgren \& K.A. Janes (L. Davis Pr., Schenectady), p. 27

Lucke, P.B. 1974, ApJS, 28, 73.

Lucke, P.B., \& Hodge, P.W. 1970, AJ, 75, 171 (LH).

Martin, N., Prévot, L., Rebeirot, E., \& Rousseau, J. 1976, A\&A, 51, 345.

Mathis, J.S., Rumpl, W., \& Nordsieck, K.H. 1977, ApJ, 217, 425.
Maucherat-Joubert, M., Lequeux, J., \& Rocca-Volmerange, B. 1980, A\&A, 86, 299.

McGee, R.X., Brooks, J.W., \& Batchelor, R.A. 1972, Aust. J. Phys., 25,581 .

Meaburn, J. 1980, MNRAS, 192, 365.

Murthy, J., \& Henry, R.C. 1994, ApJ, 448, 848.

Nandy, K., Morgan, D.H., Willis, A.J., Wilson, R., \& Gondhalekar, P.M. 1981, MNRAS, 196, 955.

Nordsieck, K.H., Marcum, P., Jaehnig, K.P., \& Michalski, D.E. 1993, Proc. SPIE, 2010, 28.

Nordsieck, K.H., Cole, A.A., Gibson, S.J., \& Harris, W.M. 1996, BAAS, 28, 757 .

Oestreicher, M.O., \& Schmidt-Kaler, Th. 1995, A\&A, 294, 57.

Pagel, B.E.J., Edmunds, M.G., Fosbury, R.A.E., \& Webster, B.L. 1978, MNRAS, 184, 569.

Parker, J.W., Hill, J.K., Cornett, R.H., Hollis, J., Zamkoff, E., Bohlin, R.C., O'Connell, R.W., Neff, S.G., Roberts, M.S., Smith, A.M., \& Stecher, T.P. 1998, AJ, 116, 180.

Pei, Y.C. 1992, ApJ, 395, 130.

Sandage, A.R. 1961, The Hubble Atlas of Galaxies, (Carnegie Observatories: Washington), p. 38

Sanduleak, N. 1969, CTIO Contrib., 89.

Sasseen, T.P., \& Deharveng, J.-M. 1996, ApJ, 469, 691.

Schwering, P.B.W. 1989, A\&AS, 79, 79.

Smith, A.M., Cornett, R.H., \& Hill, R.S. 1987, ApJ, 320, 609 (SCH).

Smith, A.M., Cornett, R.H., \& Hill, R.S. 1990, ApJ, 355, 746.

van den Bergh, S. 1981, A\&AS, 46, 79.

van den Bergh, S. 1984, in IAU Symp. No. 108, Structure \& Evolution of the Magellanic Clouds, eds. S. van den Bergh \& K.S. de Boer, (Reidel: Dordrecht), p. 1.

Westerlund, B.E. 1990, Astr. \& Astrophys. Rev., 2, 29.

Westerlund, B.E. 1997, The Magellanic Clouds, (Cambridge Univ. Press, Cambridge), p. 30

White, R.L. 1979, ApJ, 229, 954.

Witt, A.N., \& Gordon, K.D. 1996, ApJ, 463, 681.

Witt, A.N., Thronson, H.A., Jr, \& Capuano, J.M., Jr. 1992, ApJ, 393, 611.

Witt, A.N., Petersohn, J.K., Bohlin, R.C., O'Connell, R.W., Roberts, M.S., Smith, A.M., \& Stecher, T.P. 1992, ApJ, 395, L5.

Wood, K., \& Jones, T.J. 1997, AJ, 114, 1405.

Zaritsky, D., Harris, J., \& Thompson, I. 1997, AJ, 114, 1002. 


\section{APPENDIX}

\section{MODEL GEOMETRY}

Large OB associatons are assumed to dominate the diffuse ultraviolet radiation field of the model galaxy. The source list has been taken from the catalog of Lucke \& Hodge (1970), and associations are identified by their LH number. Their radii and fluxes have been derived for our model purposes in $\$ 2.1$. Our adopted source parameters have been listed in Table A1. Source positions are given in terms of the rectilinear model coordinate grid, where $x$ and $y$ are the east-west and north-south offsets, respectively, from the optical center of the LMC bar at $05^{\mathrm{h}} 24^{\mathrm{m}},-69^{\circ} 50^{\prime} \quad(\mathrm{B} 1950.0)$. The source radii do not replicate the true physical sizes of the LH associations, but are the effective radii of circular regions of the equivalent areas of the associations (see §2.1). 21 of the associations lie within the area observed by WISP (Paper I).

The smoothly distributed exponential disk of dust in our models has been seeded with cavities that approximate the extremely low density supergiant shells investigated by Meaburn 1980. The supergiant shells were identified by $\mathrm{H} \alpha-$ emission from their limbs, and roughly correspond to areas of low HI column density (Kim et al. 1998). While the real supergiant shells are approximately cylindrical, our code as currently implemented allows only spherical cavities. The resulting shells are overlain by the high- $z$ tail of the vertical dust distribution and thus fail to precisely reproduce the infrared morphology of the shells. The cavity parameters, with identifications from Meaburn 1980, are given in Table A2. In each case, the optical depth at $2150 \AA$ across the diameter of a cavity has been set to 0.1 (see $\S 2.2)$. Three of the shells are contained within the observed WISP field (Paper I). 
TABLE A1

Source parameters for Monte Carlo models.

\begin{tabular}{|c|c|c|c|c|c|c|c|c|c|c|c|c|c|c|}
\hline $\mathrm{LH}^{\mathrm{a}}$ & $x^{b}$ & $y^{b}$ & radius $^{\mathrm{c}}$ & flux $^{\mathrm{d}}$ & $\mathrm{LH}$ & $\mathrm{x}$ & $\mathrm{y}$ & rad. & flux & $\mathrm{LH}$ & $\mathrm{x}$ & $\mathrm{y}$ & rad. & flux \\
\hline $1^{\star}$ & 2.312 & -0.250 & 4.05 & 4.91 & 42 & -0.050 & -0.300 & 6.00 & 14.45 & 83 & -1.580 & 3.240 & 3.45 & 5.01 \\
\hline $2^{\star}$ & 2.250 & -0.200 & 2.10 & 3.40 & 43 & -0.100 & 3.750 & 4.35 & 5.75 & 84 & -1.532 & 2.380 & 4.65 & 26.46 \\
\hline $3^{\star}$ & 2.467 & 1.732 & 4.35 & 0.87 & 44 & -0.200 & 0.267 & 6.00 & 0.42 & 85 & -1.500 & 0.500 & 2.70 & 14.20 \\
\hline $4^{\star}$ & 2.467 & 2.132 & 4.35 & 6.26 & 45 & -0.200 & 3.480 & 8.40 & 12.91 & 86 & -1.500 & 1.820 & 2.55 & 0.79 \\
\hline $5^{\star}$ & 2.100 & 0.000 & 4.05 & 8.69 & 46 & -0.250 & -0.080 & 3.30 & 2.09 & 87 & -1.450 & -0.240 & 4.95 & 46.07 \\
\hline $6^{\star}$ & 2.267 & 1.932 & 4.35 & 3.98 & 47 & -0.267 & 1.380 & 4.50 & 92.73 & 88 & -1.532 & 1.732 & 2.10 & 0.46 \\
\hline 7 & 1.700 & -2.160 & 2.85 & 0.08 & 48 & -0.280 & 1.425 & 2.85 & 7.37 & 89 & -1.500 & 0.410 & 5.85 & 93.84 \\
\hline $8^{\star}$ & 1.920 & -0.250 & 6.75 & 10.39 & 49 & -0.350 & 1.250 & 2.85 & 4.87 & 90 & -1.500 & 0.200 & 2.85 & 19.17 \\
\hline $9^{\star}$ & 2.240 & 2.600 & 3.00 & 34.88 & 50 & -0.450 & -1.932 & 6.75 & 1.10 & 91 & -1.650 & 2.780 & 2.55 & 0.52 \\
\hline $10^{\star}$ & 2.230 & 2.700 & 2.85 & 8.71 & 51 & -0.550 & 1.840 & 2.55 & 2.40 & 92 & -1.600 & 1.820 & 1.80 & 0.50 \\
\hline $11^{\star}$ & 1.932 & 0.425 & 3.60 & 0.40 & 52 & -0.600 & 3.020 & 3.30 & 2.29 & 93 & -1.580 & 0.020 & 1.80 & 9.77 \\
\hline $12^{\star}$ & 1.950 & 0.750 & 6.00 & 21.82 & 53 & -0.650 & 3.150 & 6.30 & 2.75 & 94 & -1.550 & -0.080 & 1.80 & 28.18 \\
\hline $13^{\star}$ & 2.150 & 2.650 & 2.10 & 2.51 & 54 & -0.637 & 1.820 & 2.55 & 16.60 & 95 & -1.720 & 2.900 & 3.00 & 2.29 \\
\hline $14^{\star}$ & 2.100 & 2.732 & 2.25 & 0.12 & 55 & -0.637 & 1.680 & 4.50 & 1.82 & 96 & -1.580 & -0.040 & 7.95 & 151.84 \\
\hline $15^{\star \dagger}$ & 2.020 & 3.332 & 5.25 & 3.78 & 56 & -0.750 & -2.175 & 4.35 & 0.44 & 97 & -1.650 & 0.040 & 2.85 & 10.71 \\
\hline $16^{\star}$ & 1.350 & 0.250 & 2.55 & 1.83 & 57 & -0.680 & 0.070 & 3.00 & 2.19 & 98 & -1.620 & -0.050 & 2.10 & 4.68 \\
\hline $17^{\star}$ & 1.312 & 0.200 & 2.55 & 0.69 & 58 & -0.670 & 0.562 & 4.35 & 34.67 & 99 & -1.620 & 0.220 & 2.85 & 1.91 \\
\hline 18 & 1.250 & -1.037 & 5.55 & 2.86 & 59 & -0.760 & -0.450 & 4.35 & 1.32 & 100 & -1.720 & 0.280 & 6.00 & 25.12 \\
\hline $19^{\star}$ & 1.467 & 1.867 & 5.55 & 6.18 & 60 & -0.780 & 1.852 & 4.35 & 17.72 & 101 & -1.750 & -0.100 & 3.60 & 63.73 \\
\hline $20^{\star}$ & 1.267 & 0.267 & 2.10 & 0.36 & 61 & -0.780 & 0.400 & 2.55 & 12.59 & 102 & -1.900 & 1.870 & 3.60 & 1.15 \\
\hline $21^{\star}$ & 1.380 & 1.460 & 3.60 & 0.79 & 62 & -0.800 & -1.160 & 4.80 & 1.00 & 103 & -1.820 & -0.250 & 4.20 & 21.06 \\
\hline $22^{\star}$ & 1.360 & 1.650 & 3.30 & 0.52 & 63 & -0.800 & 1.900 & 2.70 & 14.07 & 104 & -1.840 & 0.000 & 3.90 & 23.19 \\
\hline 23 & 1.080 & -1.560 & 2.85 & 0.25 & 64 & -0.880 & 0.600 & 6.75 & 15.14 & 105 & -1.800 & -0.350 & 2.85 & 0.79 \\
\hline 24 & 1.100 & -1.300 & 6.90 & 11.71 & 65 & -1.050 & 2.350 & 2.25 & 1.74 & 106 & -1.880 & -0.160 & 14.40 & 52.48 \\
\hline $25^{\star}$ & 1.250 & 1.120 & 3.30 & 2.00 & 66 & -1.000 & -1.620 & 3.30 & 2.05 & 107 & -1.820 & -1.800 & 6.90 & 1.20 \\
\hline 26 & 1.050 & -1.150 & 6.30 & 17.72 & 67 & -1.050 & 0.125 & 3.30 & 5.87 & 108 & -1.920 & -0.250 & 3.00 & 2.51 \\
\hline 27 & 1.000 & 0.160 & 3.30 & 0.24 & 68 & -1.120 & 0.560 & 0.90 & 0.03 & 109 & -2.000 & 0.450 & 3.30 & 0.24 \\
\hline 28 & 0.820 & -1.820 & 5.10 & 1.82 & 69 & -1.100 & -1.600 & 4.05 & 7.43 & 110 & -1.865 & -1.880 & 3.60 & 0.60 \\
\hline 29 & 0.900 & -0.667 & 3.60 & 0.36 & 70 & -1.160 & 1.950 & 2.70 & 2.00 & 111 & -2.020 & 0.190 & 3.90 & 15.22 \\
\hline 30 & 0.900 & 0.050 & 2.55 & 0.17 & 71 & -1.160 & 0.750 & 2.55 & 1.51 & 112 & -2.180 & 1.950 & 2.85 & 0.93 \\
\hline 31 & 0.800 & 0.400 & 4.80 & 9.12 & 72 & -1.240 & 2.840 & 4.05 & 9.55 & 113 & -2.050 & 0.320 & 1.80 & 0.26 \\
\hline 32 & 0.900 & 2.080 & 63 & 3.31 & 73 & -1.160 & 0.650 & 2.55 & 0.46 & 114 & -2.200 & 1.425 & 3.00 & 1.58 \\
\hline 33 & 0.667 & 0.160 & 3.30 & 0.28 & 74 & -1.200 & 0.150 & 3.90 & 5.35 & 115 & -2.400 & 2.867 & 6.75 & 3.88 \\
\hline 34 & 0.680 & 1.960 & 4.50 & 2.75 & 75 & -1.180 & 1.767 & 2.10 & 2.63 & 116 & -2.380 & 2.020 & 4.80 & 7.24 \\
\hline 35 & 0.440 & 0.000 & 4.95 & 10.96 & 76 & -1.200 & 1.650 & 5.25 & 39.81 & 117 & -2.532 & -0.680 & 3.60 & 15.69 \\
\hline 36 & 0.550 & 1.960 & 2.10 & 1.91 & $77^{\dagger}$ & -1.420 & 2.350 & 9.60 & 37.84 & 118 & -2.600 & -0.732 & 2.85 & 2.49 \\
\hline 37 & 0.562 & 1.900 & 18 & 1.5 & 78 & -1.300 & 1.780 & 2.85 & 3.80 & 119 & -2.780 & 0.980 & 2.10 & 0.10 \\
\hline 38 & 0.562 & 1.820 & 2.70 & 1.28 & 79 & -1.300 & 1.850 & 3.90 & 1.91 & 120 & -2.820 & 1.040 & 4.50 & 0.55 \\
\hline 39 & 0.400 & -0.160 & 3.90 & 2.51 & 80 & -1.300 & -0.532 & 3.60 & 0.38 & 121 & -3.000 & 1.000 & 7.95 & 1.74 \\
\hline 40 & 0.000 & -1.820 & 2.10 & 0.13 & 81 & -1.350 & -0.300 & 4.95 & 55.39 & 122 & -3.320 & 0.960 & 4.05 & 0.55 \\
\hline 41 & 0.050 & 0.132 & 6.75 & 50.42 & 82 & -1.467 & 1.732 & 3.30 & 2.40 & & & & & \\
\hline
\end{tabular}

${ }^{a}$ Lucke-Hodge number, see $§ 2.1$.

${ }^{\mathrm{b}}$ Offsets in degrees from $5^{\mathrm{h}} 24^{\mathrm{m}},-69^{\circ} 50^{\prime}$ (B1950.0), increasing north and west.

${ }^{\mathrm{c}}$ Radii in arcmin, see $\S 2.1$.

${ }^{\mathrm{d}} 10^{-12} \mathrm{erg} \mathrm{s}^{-1} \mathrm{~cm}^{-2} \AA^{-1}$

${ }^{\dagger}$ Irregular, nonspherical morphology.

${ }^{\star}$ Within observed WISP field. 
TABLE A2

Cavity parameters for Monte Carlo models.

\begin{tabular}{crrr}
\hline \hline \multicolumn{1}{c}{ Shell $^{\mathrm{a}}$} & \multicolumn{1}{c}{$\mathrm{x}^{\mathrm{b}}$} & \multicolumn{1}{c}{$\mathrm{y}^{\mathrm{b}}$} & radius $^{\mathrm{c}}$ \\
\hline LMC-1 $^{\star}$ & 2.025 & 3.325 & 24.0 \\
LMC-2 & -2.225 & -0.475 & 30.0 \\
LMC-3 & -1.075 & 0.350 & 34.5 \\
LMC-4 & -1.100 & 2.375 & 40.5 \\
LMC-5 & -0.600 & 2.900 & 27.0 \\
LMC-6 & 1.950 & 0.575 & 21.0 \\
LMC-7 $^{\star}$ & 2.125 & -0.250 & 27.0 \\
LMC-8 & 1.075 & -1.225 & 30.0 \\
LMC-9 & -0.725 & -1.775 & 30.0 \\
\hline
\end{tabular}

${ }^{a}$ From Meaburn (1980), see $\S 2.2$.

${ }^{\mathrm{b}}$ Offsets in degrees from $5^{\mathrm{h}} 24^{\mathrm{m}}$, $-69^{\circ} 50^{\prime}$ (B1950.0), increasing north and west.

${ }^{\mathrm{c}}$ Radii in arcminutes, see $\S 2.2$.

${ }^{\star}$ Within observed WISP field. 